\title{
QuantiDOPA: A Quantification Software for Dopaminergic Neurotransmission SPECT
}

\author{
A. Niñerola , B. Marti- ${ }^{-}$O. Esteban , X. Planes , A.F. Frangi , M.J. Ledesma-Carbayo \\ A. Santos , A. Cot , F. Lomeña , J. Pavia , and D. Ros
}

\begin{abstract}
Quantification of neurotransmission Single-Photon Emission Computed Tomography (SPECT) studies of the dopaminergic system can be used to track, stage and facilitate early diagnosis of the disease. The aim of this study was to implement QuantiDOPA, a semi-automatic quantification software of application in clinical routine to reconstruct and quantify neurotransmission SPECT studies using radioligands which bind the dopamine transporter (DAT). To this end, a workflow oriented framework for the biomedical imaging (GIMIAS) was employed. QuantiDOPA allows the user to perform a semiautomatic quantification of striatal uptake by following three stages: reconstruction, normalization and quantification. QuantiDOPA is a useful tool for semi-automatic quantification in DAT SPECT imaging and it has revealed simple and flexible.
\end{abstract}

Keywords-Quantification, dopaminergic system, Parkinson's disease, SPECT, GIMIAS.

\section{INTRODUCTION}

Parkinson's disease is a neurological disorder associated with the loss of dopaminergic neurons. A number of ${ }^{99 m} \mathrm{TC}$ agents and ${ }^{123} I$-agents have been developed for SinglePhoton Emission Computed Tomography (SPECT) imaging to study presynaptic dopamine transporter (DAT) binding. Quantification of DAT SPECT imaging can be used to discriminate parkinsonian syndromes from other movement disorders. Quantification also facilitates an early diagnosis of the disease [1], to follow up its progression [2] and assess the effects of treatment strategies [3].

In clinical routine there is a need for quantification methods of DAT SPECT studies. This work deals with the implementation of QuantiDOPA, a semi-automatic quantification software for dopaminergic neurotransmission SPECT studies.

\section{MATERIAL AND Methods}

This medical application was developed using GIMIAS [4], a workflow oriented framework for the biomedical imaging and modeling of prototypes in the context of the Virtual Physiological Human. It is an open source framework distributed under a BSD license, developed in C++ and is based on robust open source libraries such as VTK, ITK, MITK, DCMTK, NETGEN and TETGEN, among others.

GIMIAS relies on these libraries for visualization, user interaction, data access, image and mesh processing, and is designed to easily integrate proprietary algorithms and new libraries that broaden the functions of the framework. GIMIAS is currently collaborating to develop and adopt standards that could favour interoperability with similar software in the context of the VPHNoE [5]. It also collaborates with CTK [6] (Common Toolkit), an international effort to develop interoperable software. The goal of GIMIAS is to reduce the time needed to develop a clinical prototype.

Each application implemented on GIMIAS, named plug-in, represents an specific process.

QuantiDOPA is a GIMIAS plug-in whose goal is to obtain from the gammacamera projections the striatum quantified value. To this end three modules have been developed: reconstruction, normalization and quantification.

\section{A. Reconstruction}

This is an optional module that allows us to standardize the reconstruction protocol of projections from different gammacameras. Preprocessing of projections with different smoothing filters can be performed before reconstruction. Currently, the reconstruction method is based on the filtered backprojection (FBP) algorithm with different smoothing filters.

\section{B. Normalization}

The reconstructed image is normalized to a SPECT template, using two separate steps. In the first step, a 9 parameters $3 \mathrm{D}$ affine transformation is used. The second step provides a fine adjustment of the striatum separately for each hemisphere. This is achieved by calculating the local 
correlation coefficient [7] between the reconstructed striatum and the SPECT template striatum. This procedure is done for each hemisphere.

The software allows working with a database of different SPECT templates.

\section{Quantification}

In order to calculate the mean activity values on the normalized SPECT image, QuantiDOPA allows us to select different regions of interest (ROIs). These ROIs were defined following two different approaches which are commonly used in nuclear medicine departments:

- Standardised 3D ROIs map, consisting on an automatic volumetric definition of the ROIs based on the morphology of the striatum according to the Automated Anatomical Labelling (AAL) map [8].

- 3D cylindrical ROIs [9] placed on the striatum area by the user. Eight ROIs are considered, one in the caudate and three in the putamen of each hemisphere. In order to facilitate cylinders placement, a 2D image consisting of the sum of eight central slices of the reconstructed image is provided.

Mean activity values are obtained in ROIs located in the striatum and in a background region to obtain the Specific Uptake Ratio (SUR), a parameter that quantifies the specific radioligand uptake in the striatal volume. SUR is defined as:

$$
S U R=\frac{S-B}{B}
$$

where S and B are the concentration activity in the striatum and in a background reference region of non-specific uptake, respectively.

After calculation, the global SUR value in the striatum is displayed on the screen. The individual SUR values in each ROI allows us to obtain the specific uptake in each of the four striatal regions (caudate and putamen for both hemispheres). All this information is stored in a data file.

Figure 1 schematically shows the workflow of the entire image processing included in QuantiDOPA.

\section{RESULTS}

QuantiDOPA allows the user to perform a semi-automatic quantification of striatal uptake by following the three stages mentioned above:

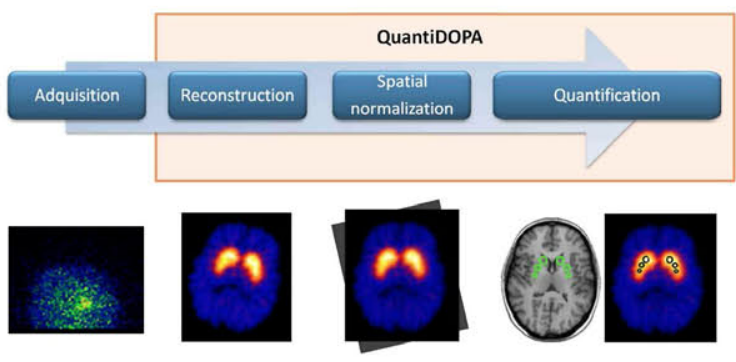

Fig. 1 Image processing workflow

\section{A. Reconstruction}

In this module the user only needs to check the parameters related to the acquisition and reconstruction, in order to run the FBP reconstruction.

Figure 2 shows and example of a reconstructed image using a 2D Butterwoth filter.
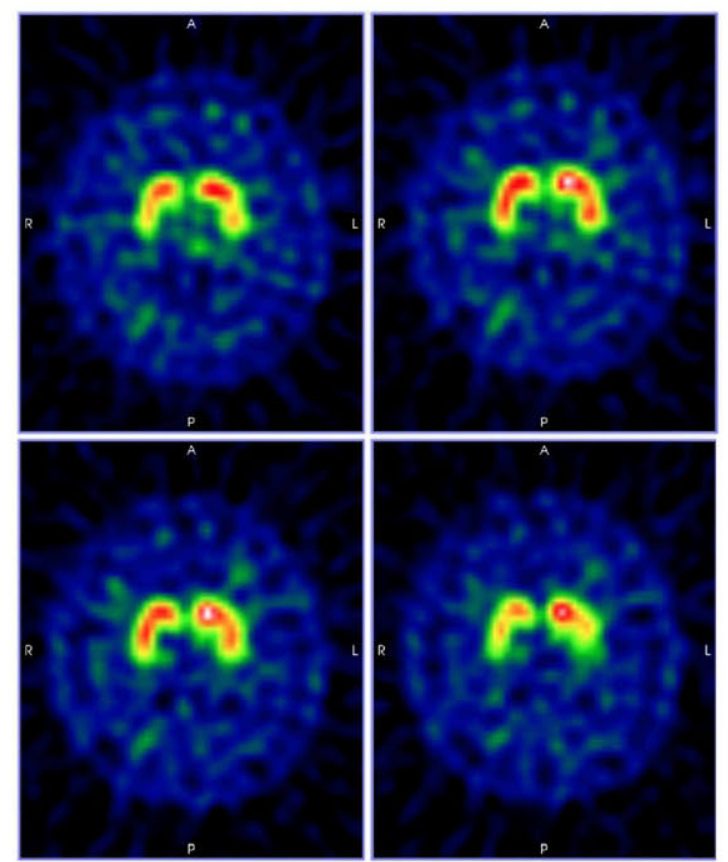

Fig. 2 Reconstructed SPECT image

\section{B. Normalization}

The image obtained in the previous module is automatically set as an input data for the normalization module. Before starting the normalization process, the user selects which template wants to normalize to.

Figure 3 shows axial, sagittal, coronal and 3D views of the SPECT study displayed in figure 2 , after the two normalization steps. 

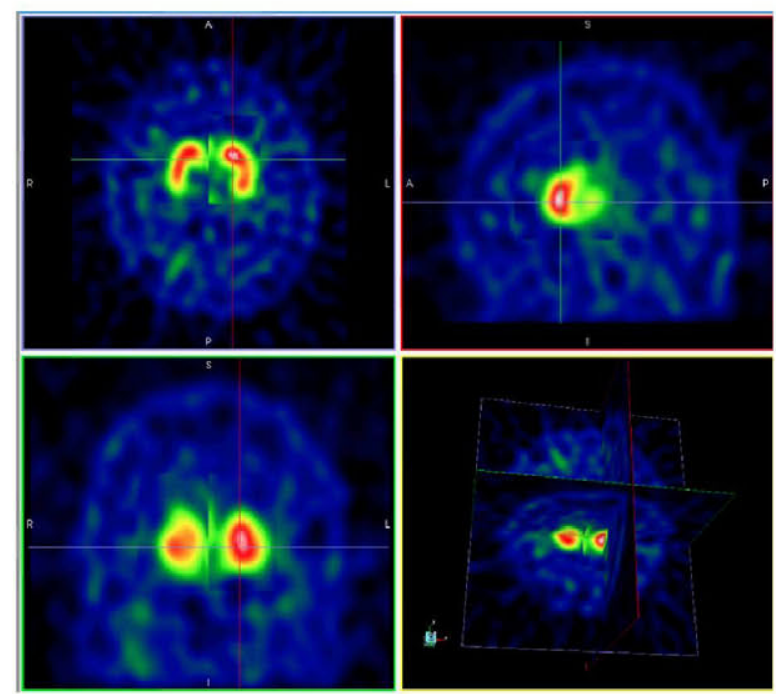

Fig. 3 Axial (top left), sagittal (top right), coronal (bottom left) and 3D view (bottom right) of the normalized SPECT image

\section{Quantification}

The normalized SPECT image obtained in the previous stage, is set as the input data of the quantification module.

ROIs based on AALs are automatically placed on the normalization study as shown in figure 4 . In the case of cylinders, the user is allowed to slightly modify the positions and to change the radius and height. Figure 5 shows a spatially normalized SPECT study with cylindrical ROIs on the striatum and the background reference region. The sum of central slices of the reconstructed image with cylindrical ROIs is also displayed.

QuantiDOPA is being used in a clinical trial to obtain SUR values from healthy volunteers in six Spanish hospitals.

\section{CONCLUSIONS}

QuantiDOPA is a useful tool for semi-automatic quantification in DAT SPECT imaging and it has revealed simple and flexible.

\section{ACKNOWLEDGEMENTS}

This work was supported in part by Spain's Ministry of Science \& Innovation (SAF2009-08076), CDTI-CENIT (AMIT project), Fondo de Investigaciones Sanitarias (PI1200390) and CIBER-BBN (MIND-t project).
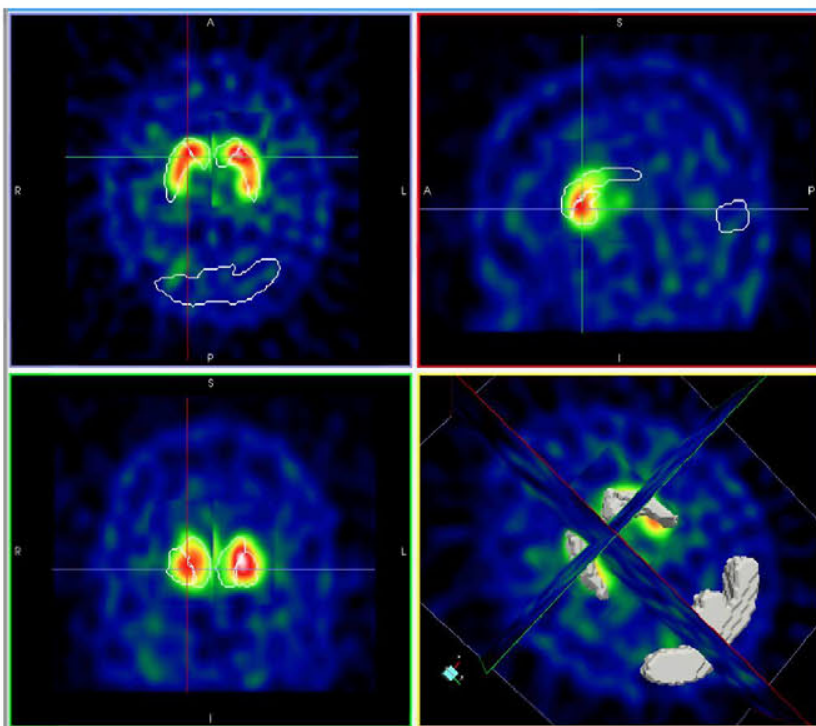

Fig. 4 Axial (top left), sagittal (top right), coronal (bottom left) and 3D view (bottom right) of the normalized SPECT image with AALs ROIs
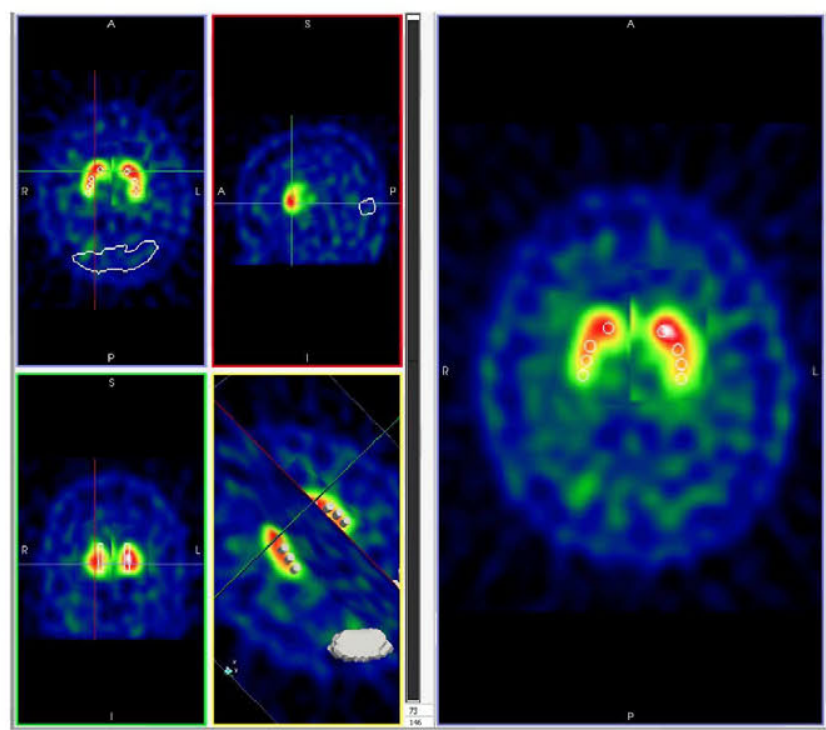

Fig. 5 Axial (top left), sagittal (top right), coronal (bottom left) and 3D view (bottom right) of the normalized SPECT image with cylindrical ROIs

\section{REFERENCES}

1. Booij J, Tissingh G, Winogrodzka A, Royen EA. Imaging of the dopaminergic neurotransmission system using single-photon emission tomography and positron emission tomography in patients with parkinsonism Eur J Nucl Med. 1999;26:171-182.

2. Seibyl JP, Marek K, Sheff K, et al. Test/retest reproducibility of iodine123-betaCIT SPECT brain measurement of dopamine transporters in Parkinson's patients J Nucl Med. 1997;38:1453-1459.

3. Stoof JC, Winogrodzka A, Muiswinkel FL, et al. Leads for the development of neuroprotective treatment in Parkinson's disease and brain imaging Eur J Pharmacol. 1999;375:75-86. 
4. Larrabide I, Omedas P, Martelli Y, et al. GIMIAS: An Open Source Framework for Efficient Development of Research Tools and Clinical Prototypes Lecture Notes in Computer Science. 2009;5528:417426.

5. Virtual Physiological Human Network of Excellence at http://www.vphnoe.eu

6. Common Toolkit at http://www.commontk.org

7. Ros D, Espinosa M, Setoain JF, Falcn C, Lomea FJ, Pava J. Evaluation of algorithms for the registration of 99Tcm-HMPAO brain SPET studies Nucl Med Communications. 1999;20:227-236.

8. Tzourio-Mazoyer N, Landeau B, Papathanassiou D, et al. Automated Anatomical Labeling of Activations in SPM Using a Macroscopic Anatomical Parcellation of the MNI MRI Single-Subject Brain NeuroImage. 2002;15:273-289.
9. Walker Z, Costa DC, Walker RWHH, et al. Differentiation of dementia with Lewy bodies from Alzheimers disease using a dopaminergic presynaptic ligand $J$ Neurol Neurosurg Psychiatry. 2002;73:134-140.

Author: Aida Niñerola Baizán

Institute: GIBUB, Universitat de Barcelona - CIBER-BBN

Street: Casanova 143 - Planta 5 (Ala Nord)

City: Barcelona

Country: Spain

Email: aninerola@ub.edu 